\title{
INFLUENCIA DEL NIVEL COGNOSCITIVO EN EL DESEMPEÑO EN EL LABORATORIO
}

TAGLIAFERRI, A.A., URE, J.E. y DIBAR URE, M.C. Instituto de Física. Universidade Federal Fluminense. Brasil.

\section{SUMMARY}

In a laboratory session which integrated the concepts of diffraction, interference and diffraction grating, planned for students in their third semester of elementary physics we detected that a relatively large percentage of students did not adequately separate nor control the intervening variables. We then correlated this cognitive ability to the performance in one section of the laboratory work which was evaluated separately.

\section{INTRODUCCION}

En un trabajo anterior (Dibar Ure y Queiroz 1977) habiamos constatado que un alto porcentaje de alumnos de ingeniería, física y química no controlan adecuadamente todas las variables, esto es, al estudiar la dependencia de un cierto fenómeno con una variable dada, no mantenían constante las otras. En particular, de un grupo de 68 alumnos de física que cursaban la primera física elemental, $44 \%$ con $s=6 \%$ (Garrett, 1971) no controló las variables para estudiar su influencia una a una. Habiamos usado para el estudio mencionado una versión escrita de la prueba de flexibilidad de la varetas (Inheider y Piaget, 1955). Piaget asocia esta habilidad con la aparición de las operaciones formales, o sea del razonamiento hipotético-deductivo, fundamental para la comprensión de las ciencias.

Al realizar una práctica de óptica con alumnos que cursaban el 3er semestre de física básica nos sorprendió encontrar evidencias de que fallas en este control de variables estaban interfiriendo con la realización y comprensión del fenómeno observado. Como esto indicaria que este problema persistía un año después del ingreso a la Universidad nos propusimos investigarlo sobre la marcha.

Describiremos aquí esta investigación realizada con un grupo relativamente pequeño de alumnos, así como la práctica de óptica durante la cual realizamos este estudio pues ella fue pensada también con base en la teoria de Piaget, dando valor a la actividad del alumno. Pensamos que por esta característica y por dejar que el alumno planee la experiencia a realizar pudimos detectar y estudiar los problemas de razonamiento.

\section{EL CONTEXTO DE LABORATORIO}

En los cursos de óptica básica, el alumno realiza, en general, experiencias de interferencias y difracción separadas. Esto es, difícilmente se realizan experiencias con dos ranuras (Experiencias de Young) en la cual se lleve en consideración el ancho de las ranura: por el contrario, las ranuras se hacen lo suficientemente estrechas como para que el máximo central de difracción ocupe casi todo el campo de visión.

Luego de la experiencia de dos ranuras, el alumno pasa a realizar experiencias con redes de difracción, en las cuales tiene 300,400 o más ranuras por $\mathrm{mm}$ sin haber visto el resultado de ir aumentando gradualmente el número de ranuras.

Con la idea de evitar este inconveniente, nos propusimos realizar con alumnos del cuarto semestre de Física General del curso de Fisica de la Universidad Federal Fluminense, una experiencia en la cual los conceptos de interferencia, difracción y red de difracción fuesen gradualmente presentados en forma experimental, dando gran importancia a la participación de los alumnos. Esta participación nos parece fundamental en un aprendizaje que apele al razonamiento y lleve a una comprensión profunda del problema planteado.

Esta experiencia nos pareció entonces particularmente apropiada por varias razones, entre las cuales vale la pena destacar:

a) El alumno tiene la posibilidad de trabajar con un minimo de instrucciones, ya que la observación del fenómeno, como sucede generalmente en óptica, es directo y él mismo puede decidir en medir las diferentes 
figuras que aparecen en la pantalla.

b) Dejando de lado las complicaciones que puedan aparecer en la teoría, es posible trabajar, con tres variables experimentales: ancho de las ranuras, separación entre ranuras y número de ranuras. Ofreciendo entonces al alumno una gran variedad de combinaciones, él puede descubrir y estudiar el efecto de variar cada una de ellas independientemente y (es justamente aquí donde aparecieron problemas).

c) Permite la realización de una experiencia del tipo previsión verificación en la que el propio alumno verifica sus conclusiones.

\subsection{Material utilizado}

Las ranuras empleadas en este trabajo fueron obtenidas fotográficamente, utilizando pelf́cula de alto contrastre (Tagliaferri, 1983). Fueron dibujadas sobre cartulina blanca, usando tinta china negra, una serie de líneas paralelas de ancho de $3 \mathrm{~mm}$ y separación de 3 , $6 \mathrm{~mm}$... El número de estas líneas en cada hoja de cartulina fue variado entre I y 40 .

El objetivo normal de una cámara fotográfica, $\mathrm{f}-50$ $\mathrm{mm}$, produce una reducción en el negativo de 45 veces para un objeto a $3 \mathrm{~m}$, de 90 veces para un objeto a 6 $\mathrm{m}$, y así sucesivamente. Aprovechando este hecho, fueron fotografiados los dibujos previamente realizados obteniéndose asi lineas transparentes sobre fondo negro en los negativos, los que pasan a ser utitizados como ranuras. Las dimensiones de estas ranuras son del orden de décimas o centésimas de mm, su costo es muy bajo y es muy fácil obtener las transparencias con las dimensiones deseadas y en cantidades que permitan a cada alumno hacer experiencias participando de un grupo pequeño.

La experiencia fue realizada utilizando un laser de $\mathrm{He}-$ Ne de $0,5 \mathrm{mw}$ de potencia; esto permite proyectar las figuras en una pantalla a $1 \mathrm{~m}$ de distancia de las ranuras, posibilitando asi su fácil visualización y medición.

\subsection{Realización de la experiencia}

La experiencia fue realizada en tres partes. El primer día se trabajó en forma totalmente cualitativa. Los alumnos observaron las figuras producidas por diferentes transparencias, guiados sólo por tres preguntas: qué observa cuando varía la distancia de separación entre las ranuras (b)? cuándo varía el ancho (a)? y el número de ranuras (N)? Usamos para este fin transparencias de $a=0.014 \mathrm{~mm}, 0.034 \mathrm{~mm}$ y $0.042 \mathrm{~mm}, \mathrm{~b}=$ $0.049 \mathrm{~mm}, 0.028 \mathrm{~mm}$ (con un error estimado en \pm $0.007 \mathrm{~mm}$ en todos los casos), y $\mathrm{N}$ de $1,2,3,4,5,10$, 20,30 , ranuras; con estas transparencias se consigue ver claramente el nacimiento de una red de difracción. Fue posible también observar la influencia del ancho de las ranuras.

En el segundo día se presentó y discutió la teoria correspondiente, cotejando continuamente con lo que ha bía sido observado en el laboratorio.

Finalmente, en el tercer día se volvió al laboratorio y la tarea de los alumnos fue identificar una transparencia que contenía un número desconocido de ranuras, de ancho y separación también desconocido. Usamos para estas transparencias los siguientes valores: $N=$ $3,465, a=0.014 \mathrm{~mm}$ y $b=0.042 \mathrm{~mm}$.

EI diseño de la tercera parte de la experiencia está dentro del espíritu de las experiencias de previsiónverificación propuesta por Neldeski (L. Nedelski,1965). En estos experimentos, la meta de los alumnos es comprender los fenómenos relevantes que les permitan predecir los resultados de una experiencia de prueba. El paso final es la verificación experimental de la predicción, consistente en comparar con los datos de construcción de la transparencia. La comprensión del fenómeno pasa por un estudio cualitativo antes de una discusión teórica.

Estamos de acuerdo con Nedelski en que ueste es un desafío explícito a la habilidad de los alumnos. (La experiencia) se realiza como una genuina búsqueda de conocimiento experimental o teórico que (el alumno) posee».

\section{EL PROBLEMA DE LAS ESTRUCTURAS COGNOSCITIVAS DE LOS ALUMNOS}

Como vimos en la primera parte, el problema experimental propuesto a los alumnos consistió en estudiar la influencia de 3 parámetros en las figuras de difracción.

Al detectar que muchos de ellos parecian no controlar bien estos 3 parámetros, decidimos investigar esto usando un problema escrito que nos permitiria estudiar más claramente como estaban controlando las variables. Este problema (ver apéndice) simulaba una situación experimental muy parecida a la que habian realizado la clase anterior: dadas las características de 8 transparencias con diferentes valores de a, b, y $\mathrm{N}$, se les pide que elijan cuáles usar para estudiar la dependencia de la figura de difracción con $\mathrm{a}, \mathrm{b}, \mathrm{y} \mathrm{N}$, en las preguntas $a_{1}, b_{1}, c_{1}, y$ también se les pregunta qué observaron en cada caso en el laboratorio (preguntas $a_{2}, b_{2}, c_{2}$ ).

Pensamos que la resolución de un problema escrito basado en situaciones ya trabajadas en el Jaboratorio se aproxima de la entrevista clínica utilizada por la escuela de Ginebra (Inhelder 1975) en la cual el alumno responde a las preguntas del entrevistador mientras manipula el material provisto.

Encontramos que $35 \%(\mathrm{~s}=11 \%)$ de nuestros 20 alumnos no controló las variables que no estaban estudian. do, mientras que $20 \%$ erró en el item $c_{1}$ (o sea que al estudiar el efecto de $N$ no controlaron que a y $b$ fuesen constantes).

Si bien hubo una mayoría (si no tenemos en consideración los que erraron el item $c_{1}$ ) en el control de las 
variables de los alumnos del $1^{\circ}$ semestre a los del $4^{\circ}$ semestre, encontramos muy alarmante el hecho que un número tan alto de alumnos del $4^{\circ}$ semestre falle en una habilidad tan elemental.

La persistencia de esta dificultad desde Física I hasta Física IV, nos marca la necesidad de realizar permanentemente actividades de laboratorio que ejerciten, en su ejecución, ésta y otras actividades básicas del razonamiento. Esta fue una propuesta que nos llevó a mo* dificar la manera de enfocar la Física I (Queiroz y Di. bar Ure, 1981) pero si bien no es de extrañar que un semestre no llegue a modificar el nivel operatorio de los alumnos, da una seria preocupación encontrar este porcentaje tan alto de errores tres semestres después.

Las preguntas sobre la observación cualitativa $\left(a_{2}, b_{2}\right.$, $c_{2}$ ) que acompañan al problema de control de varia ble fueron corregidas en forma completamente independiente por otro profesor que dio a cada alumno una nota entre cero y tres.

Esto nos permitió estudiar la influencia del nivel cognoscitivo (reflejado en el control de variables) en la comprensión de lo observado en el laboratorio. El pro* medio de las notas obtenidas por los alumnos que no controlan variables en estas preguntas es $1,14(s=0,37)$ mientras que del resto es $1,73(s=0,24)$. Usando la prueba bilateral $t$ de Student (Spiegel, 1977) para comparar los promedios como la más adecuada debido al tamaño de nuestra muestra, obtenemos $t=4,02$, con 18 grados de libertad. Por lo tanto podemos rechazar la hipótesis nula (que los promedios obtenidos en la observación son iguales) en un nived de significación $<0,001$,

Este resultado vincula directamente la capacidad de separar y controlar variables que intervienen en una experiencia con la descripción y comprensión de las observaciones de laboratorio. Es interesante aclarar que esto ocurre a pesar de que cuando fue realizado el laboratorio el material entregado presentaba las varia. bles prácticamente separadas pues las transparencias contenian varias series de ranuras, por ejemplo una serie con 3 anchos (b) con el mismo número de ranuras (N) y la misma distancia (a) en una única transparencia y asi sucesivamente.

Seguramente asociado a esta misma dificultad de es. tudiar el fenómeno separando y controlando las variables, notamos que varios alumnos durante el laboratorio y hasta en la evaluación final del trabajo, se re ferian al estudio de las transparencias previstas (4) co. mo a «cuatro experiencias realizadas», como si no pudiesen ver la experiencia en su totalidad como la realización de una experiencia que estudia la influencia de 3 variables en un fenómeno, si no que se centraban en la descripción del material tal como se les presentaba. Este comportamiento también sería característico de los alumnos que usan esquemas típicos del periodo de las operaciones concretas.
Ya el informe de laboratorio, hecho en grupo, mostró muy buenos resultados, sin grandes errores. Es de notar que los grupos (de 3 ó 4 personas), formados libremente, eran bastante similares; todos tenían por lo menos algún componente que controlaba variables y por lo menos 1 componente que no controlaba variables o erraba en la última pregunta del problema.

\section{EVALUACION DE LOS ALUMNOS SOBRE LA PRACTICA ACTIVA}

Los alumnos respondieron anónimamente a ta pregunta: qué les gustó y qué no les gustó de las últimas 2 experiencias de laboratorio.

Aparte de algunos factores prácticos que incomodaron, como mucha gente en una sala, no tener posibilidad de controlar la luz individualmente, y tiempo limitado, todas las evaluaciones fueron positivas, muchas indicando específicamente que este laboratorio fue «de los más lindos y provechosos» y que "consiguió juntar cosas que habian quedado dispersas en la cabeza después de haber leído la teoria). Un alumno sugirió que todas las clases de física fuesen ast.

A algunos les gustó más la parte cualitativa. Varios indicaron que les agradó la «relación» (sic) conjunta de explicación experimentación porque hace que la fijación de aprendizaje sea rápida y sólida».

\section{CONCLUSIONES}

La medida realizada aún cuando no muy precisa por tratarse de una muestra pequeña nos mostró que un porcentaje, que consideramos alto, de alumnos no trabaja aún correctamente, controlando las variables que intervienen, ni puede describir correctamente lo observado en el laboratorio.

Este porcentaje ha variado poco desde Física I, por lo tanto nos indica que se debe dar más importancia a tratar de desarroliar este tipo de habilidades, que son indispensables para un buen desempeño y comprensión del iaboratorio, a la vez que estarian vinculadas según la epistemología genética a otras capacidades igualmente importantes asociadas al razonamiento hipotético deductivo (trabajo con proporciones y capacidad de pro. bar hipótesis).

Pudimos comprobar efectivamente que hay una diferencia significativa en la comprensión de los resulta. dos entre los alumnos que controlan variables y aquellos que no controlan variables.

Es importante resaltar la importancia de este resultado que vincula directamente la capacidad de separar y controlar las variables que intervienen en una experiencia de laboratorio, con la correcta descripción de los fenómenos observados en la misma. 
Corresponde aclarar que el material utilizado en el laboratorio, esencialmente las transparencias, permitía separar fácilmente las variables, que fue precisamente realizado por los diferentes grupos y que se manifiesta en la redacción de los informes. Sin embargo el problema escrito nos muestra que pese a que planeamos una práctica con el objetivo de identificar la influencia del ancho, separación y número de ranuras en la imagen proyectada, y pese a que el trabajo grupal fue realizado básicamente de acuerdo a lo planeado, existe un número apreciable de alumnos que no consigue aislar la influencia de una variable y en consecuencia, cuando trabaja fuera del grupo, no alcanza a comprender la experiencia.

Esto nos sugiere que a pesar de que se prepare el material para ayudar al alumno, el nivel cognitivo influye, como planteado por la escuela de Piaget (Inhelder y Piaget, 1955) en la forma de trabajar en el laboratorio. Según la psicología genética, un alumno de nivel de operaciones formales se coloca de entrada frente a los resultados posibles, separa variables, plantea hipótesis y las pone a prueba. Por el contrario, un alumno que aún se encuentra en las operaciones concretas se limita a hacer una lectura de los casos que se presentan sin poder, muchas veces, generalizar correctamente.
Apéndice (traducido del portugués) Suponga que en el laboratorio le entregan las siguientes ranuras (hechas fotográficamente) para estudiar interferencia y difracción.

transparencias
(1) $\mathrm{a}=0.050 \mathrm{~mm}$
$\mathrm{b}=0.049 \mathrm{~mm}$
$N=4$
(2) $\mathrm{a}=0.042 \mathrm{~mm}$
$\mathrm{b}=0.49 \mathrm{~mm}$
$N=2$
(3) $a=0.034 \mathrm{~mm}$
$\mathrm{b}=0.43 \mathrm{~mm}$
$\mathrm{N}=2$
(4) $a=0.014 \mathrm{~mm}$
$\mathrm{b}=0.028 \mathrm{~mm}$
$\mathrm{N}=10$
(5) $\mathrm{a}=0.042 \mathrm{~mm}$
$\mathrm{b}=0.252 \mathrm{~mm}$
(6) $a=0.014 \mathrm{~mm}$
$\mathrm{b}=0.028 \mathrm{~mm}$
$\mathrm{N}=2$
(7) $a=0.028 \mathrm{~mm}$
$\mathrm{b}=0.03 \mathrm{~mm}$
$\mathrm{N}=5$
$N=4$
(8) $\mathrm{a}=0.060 \mathrm{~mm} \quad \mathrm{~b}=0.49 \mathrm{~mm}$
$N=2$

Responda a las siguientes preguntas

$a_{\mathrm{f}}$ ) ¿Qué transparencias elegiría usted para estudiar el efecto de $a$ ?

a) ¿Qué observó en el laboratorio al variar a?

$\left.b_{1}\right)$ ¿Qué transparencias elegiria usted para estudiar el efecto de $b$ )?

$\left.b_{2}\right)$ ¿Qué observó en el laboratorio al variar b?

$c_{\ddagger}$ ) ¿Qué transparencias elegiria usted para estudiar el efecto de N?

c) ¿Qué observó en el laboratorio al variar N?

$\mathrm{a}=$ distancia entre las ranuras

$\mathrm{b}=$ ancho de las ranuras

$\mathrm{N}=$ número de ranuras

\section{REFERENCIAS BIBLIOGRAFICAS}

DIBAR URE, M.C. y QUEIROZ G.R.P., 1979, Nova tentativa para a realizaçao de un teste coletivo (IV Simposio de Ensino de Física, Rio de Janeiro).

INHELDER B., BOVET, M. y SINCLAIR, H., 1975, Aprendizaje y estructuras del conocimiento (Madrid, Morata) (Original: 1974).

INHELDER B. Y PIAGET, J., 1972, De la lógica del nino a la lógica del adolescente (Buenos Aires, Paidós) (original: 1955).

NEDELSKI, L., 1965, Science teaching and testing (N. York, Harcourt and Brace).
QUEIROZ, G.R.P. y DIBAR URE, M.C., 1981, Una experiéncia de ensino na $1^{2}$. cadeira de Física Básica na universidade Revista de Ensino de Física, Vol, 3, $\mathrm{n}^{\circ} 4$, pp. 3-12.

SPIEGEL, M.R., 1977, Estatística (São Paulo, McGraw Hill de Brasil).

TAGLIAFERRI, A.A., 1983, Construção e utilização de fendas fotográficas para o ensino de ótica (XXXV Reunião Anual da Sociedade Brasileira para o Progresso de Ciência. 18-D-19. Belém). 